Indexed by

\section{Scopus}

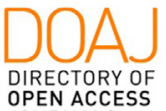

OPEN ACCESS

JOURNALS

Crossref

$\mathrm{R} \odot \mathrm{AD} \equiv$

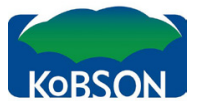

Google

\title{
MICROPARTICLE SEPARATION IN A LINEAR PAUL TRAP
}

\section{Roman Syrovatka}

Joint Institute for High Temperatures of the Russian Academy of Sciences (JIHT RAS), Moscow, Russia

\section{Vladimir Filinov}

Joint Institute for High Temperatures of the Russian Academy of Sciences (JIHT RAS), Moscow, Russia

\author{
Leonid Vasilyak \\ Joint Institute for High Tem- \\ peratures of the Russian Acad- \\ emy of Sciences (JIHT RAS), \\ Moscow, Russia
}

\section{Vladimir Pecherkin}

Joint Institute for High Temperatures of the Russian Academy of Sciences (JIHT RAS), Moscow, Russia

\author{
Lidiya Deputatova \\ Joint Institute for High Tem- \\ peratures of the Russian Acad- \\ emy of Sciences (JIHT RAS), \\ Moscow, Russia
}

\author{
Vladimir Vladimirov \\ Joint Institute for High Tem- \\ peratures of the Russian Acad- \\ emy of Sciences (JIHT RAS), \\ Moscow, Russia
}

Key words: separation, microparticles, Paul trap, charged particles doi:10.5937/jaes0-28342

\section{Cite article:}

Syrovatka, R., Filinov, V., Vasilyak, L. Pecherkin, V., Deputatova, L., Vladimirov, V. (2021) MICROPARTICLE SEPARATION IN A LINEAR PAUL TRAP, Journal of Applied Engineering Science, 19(3), 564-569, DOI:10.5937/jaes0-28342 


\title{
MICROPARTICLE SEPARATION IN A LINEAR PAUL TRAP
}

\author{
Roman Syrovatka*, Vladimir Filinov, Leonid Vasilyak, Vladimir Pecherkin, Lidiya Deputatova, \\ Vladimir Vladimirov
}

Joint Institute for HighTemperatures of the Russian Academy of Sciences (JIHT RAS), Moscow, Russia

We investigated the charged micron-sized particle separation by the alternating electric field in a linear quadrupole electrodynamic trap in open air under standard atmospheric temperature and pressure conditions (STP). In experiments we varied the amplitude of the alternating voltage supplying the electrodynamic trap and used a mixture of charged glassy carbon and alumina particles. The carried out numerical simulations and experimental results showed the mutual influence of the amplitude and frequency of the supplied to the trap electrode voltage on the separation of the different sizes particles. The typical particle charges in simulations were approximately equal to experimentally measured values obtained in a corona discharge.

Key words: separation, microparticles, Paul trap, charged particles

\section{INTRODUCTION}

Separation of polydispersed powders into fractions is an important problem for different applications. Monodispersed powders are used, for example, in research of dusty plasma [1-3], in particle image velocimetry methods for determining the velocity of liquids and gases [4], in nanotechnology [5]. Monodisperse aerosols are used in studies of Coulomb clusters [6]. We can mention the most popular ways of the particle separation like centrifuges [7], dielectrophoresis [8], photophoresis [9], optophoresis [10] or the self-organization of particles in the nodes of standing acoustic waves [11]. In mass spectrometry in vacuum conditions the linear quadrupole traps can be used as filter allowing only the ions of certain masses pass along the trap axis. There is a great interest for devices, in which a large number of charged particles of different masses can be separated. Electrodynamic Paul traps, which can provide levitation of charged particles up to hundreds of microns in size, look promising in this regard [12-15]. The confinement of particles in such traps substantially depends on the operating parameters [16-23]; therefore, by varying the frequency or the magnitude of the applied voltage, one can achieve confinement of particles of a certain type.

The aim of this work is to study the spatial separation of charged micron-sized particles in open air under STP in a linear electrodynamic quadrupole trap. The carried out numerical simulations supported experimental results and showed the mutual influence of the amplitude and frequency of the supplied to the trap electrode voltage on the separation of particles of different sizes.

\section{EXPERIMENTAL SETUP AND MATHEMATICAL DESCRIPTION OF THE PARTICLE DYNAMICS}

The electrodynamic trap consists of four cylindrical electrodes 1-4 (Fig. 1), which are supplied by an alternating electric potential $U=U_{\alpha} \sin (\omega t)$. In the linear quadrupole trap shown in Fig. 1, two additional electrodes 5 are lo- cated on the axis of the trap, to which a constant electric potential is supplied to prevent particle escaping from the trap.

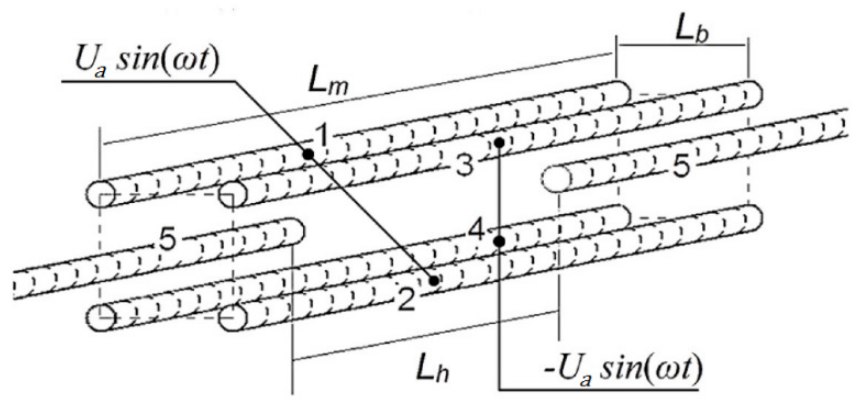

Figure 1: Scheme of a linear quadrupole electrodynamic trap: 1-4 is the trap electrodes, to which an alternating electric potential is applied, ensuring the particles confining in the radial direction; 5 is the end electrodes, to which a constant potential is applied, ensuring confining in the axial direction.

Mathematical simulation of the dynamics of a dust particle in a linear electrodynamic trap was carried out by numerically integrating the system of Newton equation:

$m_{i} \frac{d \boldsymbol{r}_{i}^{2}}{d t^{2}}=m_{i} \boldsymbol{g}-q_{i} \nabla \boldsymbol{\Psi}+\boldsymbol{F}_{f r}+\sum_{j}^{j \neq i} k \frac{q_{j} q_{i}}{r_{j i}{ }^{2}} \frac{\boldsymbol{r}_{j i}}{r_{j i}}$

where $\psi$ is the electrode electric potential of the trap, $k$ is the coefficient of proportionality in the Coulomb law, $i, j=1,2 \ldots n, n$ is the number of particles. The electric potential in the trap region was found by numerically solving the Laplace equation by the finite element method in the two-dimensional case. The computational domain was a circle with a diameter of one meter, the center of which coincided with the center of the trap. At the boundary of this region, the potential was set equal to zero. During the numerical integration of the equations of motion, the potential in a square region of 4 by $4 \mathrm{~cm}$ has been calculated. The last term in the equation (1) expresses the interparticle interactions. As can be seen from equation (2), the mass and charge are essential characteristics of 
a particle that have to influence on its dynamics in a trap. The drag force proportional to the size of the particle is also important as in test calculations, with a five-fold increase in particle size at a constant mass, the particle ceased to be confined in the trap.

Qualitative explanation of the charged particle capturing in trap is the following. If the charged particle is in a high-frequency electric field $\mathbf{E} \sin (\omega t)$, an effective force acting on it is directed towards decreasing the field strength [18]:

$$
\boldsymbol{F}=-\frac{q^{2}}{2 m\left(\omega^{2}+v^{2}\right)} \nabla \boldsymbol{E}^{2}
$$

where $q$ and $m$ is the particle charge and mass. In plasma physics, this force is called the force of high-frequency pressure. It should be noted that expression (2) is true if the viscous friction force linearly acting on the particle depends on the particle velocity $\mathbf{F}_{\mathrm{fr}}=-m \mathbf{v}$. In our case, the Stokes law describing the motion of a particle is satisfied $\mathbf{F}_{\mathrm{fr}}=-3 \pi \mu d \mathbf{v}, v=3 \pi \mu d / m$, where $\mu$ is the dynamic viscosity, $d$ is the particle diameter). The force (2) counteracts the force of gravity and ensures the levitation of the particle. It decreases with increasing frequency and ceases to confine the particle in the trap starting from a certain value. Since the confining force is proportional to $q^{2} / m$, spatial separation of particles with different values of charge and mass can be expected. To verify this assumption, a numerical experiment was carried out.

\section{EXPERIMENTAL RESULTS}

In our work, we carried out the following experiment. A mixture of two types of particles (glassy carbon with size $d=15 \div 20 \mu \mathrm{m}$ and density $\rho=1.5 \mathrm{~g} / \mathrm{cm}^{3}$ and aluminium oxide with $d=50 \div 60 \mu \mathrm{m}$ and $\rho=3.99 \mathrm{~g} / \mathrm{cm}^{3}$ ) was passed through a corona discharge, where they were charged and then injected into an electrodynamic trap operating at $U_{a}=3 \mathrm{kV}$ and $\omega=2 \pi \times f \mathrm{rad} / \mathrm{s}, f=50 \mathrm{~Hz}$. Left panel on Fig. 2 shows a photograph of the mixture of particles confined in the trap at the specified parameters. One can see more and less bright dots representing aluminium

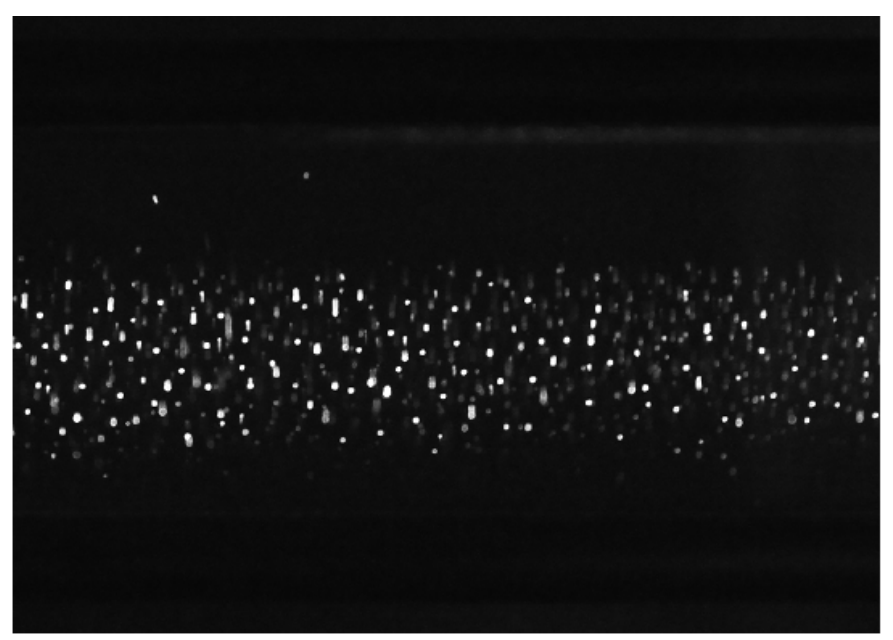

oxide and glassy carbon particles respectively. When the voltage $U_{a}$ decreases to $1.5 \mathrm{kV}$, aluminum oxide particles are deposited downward and only glassy carbon particles remain in the trap as shows right panel on Fig. 2. One can see, that two larger particles remain in the trap after voltage reduction. Perhaps it can be particles of aluminum oxide for some reason charged to a greater value or stuck together glassy carbon particles. Thus, separation of the mixture of particles of two different types has been achieved.

The most important particle parameters for its dynamics in an electrodynamic trap are charge and mass. Measurements of the particle charge, after charging in a corona discharge of positive and negative polarity, were carried out in one of our previous works [19]. The measurement results are shown in Fig. 3 for the convenience of readers. The solid lines indicate the maximum charge that can receive particle in a unipolar medium in an electric field with a strength of 10 and $20 \mathrm{kV} / \mathrm{cm}$.

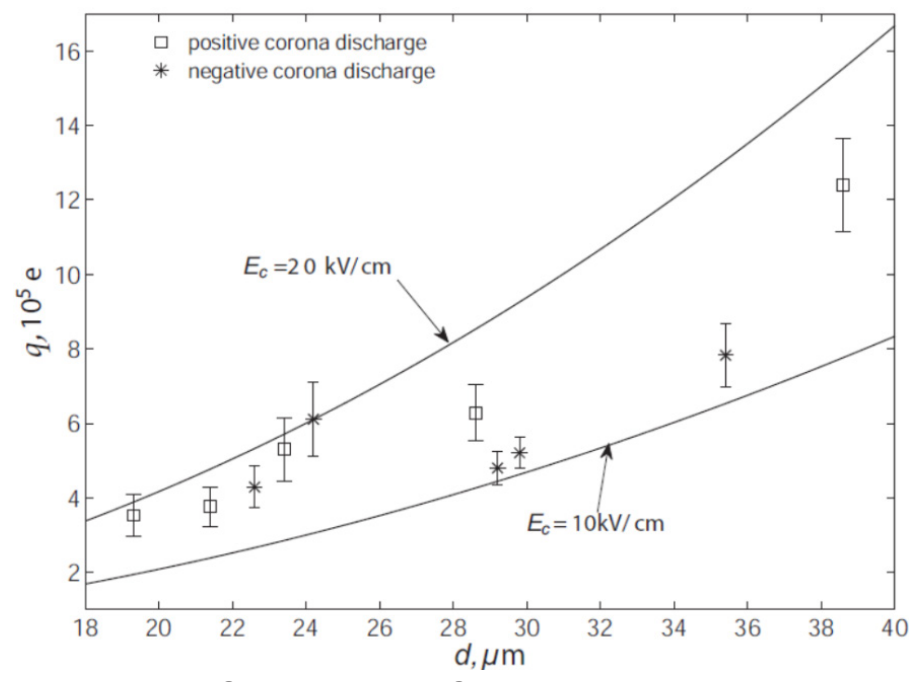

Figure 3: Charges on $\mathrm{Al}_{2} \mathrm{O}_{3}$ particles obtained in the negative (asterisks) and in the positive (squares) corona discharge. The lines indicate the maximum charge that a particle can receive in a unipolar medium in a field with a strength of 10 and $20 \mathrm{kV} / \mathrm{cm}$ [19]

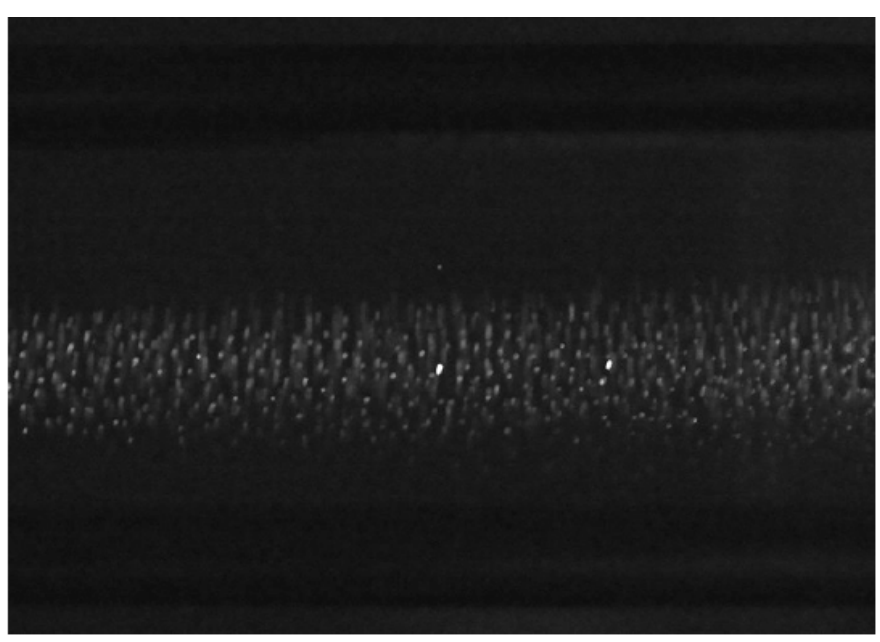

Figure 2: Left panel: The mixture of charged particles of two types injected into the trap at $U_{a}=3 \mathrm{kV}$ and $\omega=2 \pi \times 50 \mathrm{rad} / \mathrm{s}$. Right panel: particles remaining in the trap when $U_{a}$ decreases to $1.5 \mathrm{kV}$ 


\section{NUMERICAL SIMULATION}

Knowing the sizes, mass density and charges of particles captured by the trap, it is possible to carry out simulations corresponding to experimental conditions. Using the data presented in Fig. 3, the following parameters were chosen for two groups of particles of different sizes. For group 1, representing particles of aluminum oxide, the particle size $\mathrm{d}_{1}$ was $54 \mu \mathrm{m}$, and their expectation charge value $q_{\mu_{1}}$ was $2 \times 10^{6} e$, where $e$ is the elementary charge. For group 2, representing particles of glassy carbon, $d_{2}=17 \mu \mathrm{m}$ and $q_{\mu 2}=2.5 \times 10^{5} \mathrm{e}$. Since the charge a particle receives in the corona discharge region depends on the field strength, the charges of particles moving along different trajectories may be different. To consider this fact, the particle charge was set by the normal distribution with median equal to expectation charge value. Assuming a spread in particle charges of $10 \%$ of the median value, the standard deviation was set at $1.0 \times 10^{5} \mathrm{e}$ for group 1 and $1.25 \times 10^{4} e$ for group 2 . The number of particles was 10 and 90 for groups 1 and 2 respectively. As initial conditions for numerical integration of the equations of motion, the particle coordinates have been randomly assigned in horizontal and vertical directionin the range from -0.5 to $0.5 \mathrm{~cm}$ along the trap axis.

Fig. 4 shows the simulation results for particle groups 1 and 2 representing by the dots and circles respectively. On the left panel of Fig. 4, one can see that at a voltage of $3 \mathrm{kV}$, both groups of particles are confined in the trap. When the voltage decreases to $1.6 \mathrm{kV}$, only one particle of group 1 with the highest charge remains in the trap (right panel on Fig. 4). With a further decrease in voltage, only particles of group 2 remain in the trap. Thus, we obtained a correspondence between the experiment and the simulation and validated our mathematical model.

Using numerical simulations, we have also analyzed the possibility of separating particles by varying the frequency of the supply voltage. For clarity, it was decided to simulate particles of the same density, the diameter of which differs by two times. In group 1 ten particles of the size $40 \mu \mathrm{m}$ had charge of $10^{6} \mathrm{e}$, while in group 2 ninety particles were twice smaller $(20 \mu \mathrm{m})$ with charge of $3 \times 10^{5}$ $e$. The mass density for both groups was $3.99 \mathrm{~g} / \mathrm{cm}^{3}$. The amplitude of the alternating voltage at the electrodes of the trap $U_{a}$ was $2.5 \mathrm{kV}$. Fig. 5 shows the results of simulations performed at four frequencies: 42, 50, 150 and 190 $\mathrm{Hz}$. At the frequency of $42 \mathrm{~Hz}$ (Fig. 5a), smaller particles with a large value of $\mathrm{q}^{2} / \mathrm{m}$ are too strangly affected by the force of the alternating electric field and therefore fly away in different directions. Thus, only larger particles remain confined in the trap. As the frequency increases to $50 \mathrm{~Hz}$, both types of particles are confined in the trap, but there is a space gap between them and this situation remains to a frequency about of $140 \mathrm{~Hz}$. Fig. 5c shows the result of calculation at frequency of $150 \mathrm{~Hz}$. At this frequency, the force acting on the larger particles is insufficient to ensure their levitation and only smaller particles remain confined in the trap. With a further increase in frequency, small particles also cease to confine and fall out. It should be noted that the separation of particles by varying the frequency looks more preferable than by varying the voltage, since the voltage required for only particles with a lower value $\mathrm{q}^{2} / \mathrm{m}$ to remain in the trap can be greater than the breakdown voltage.
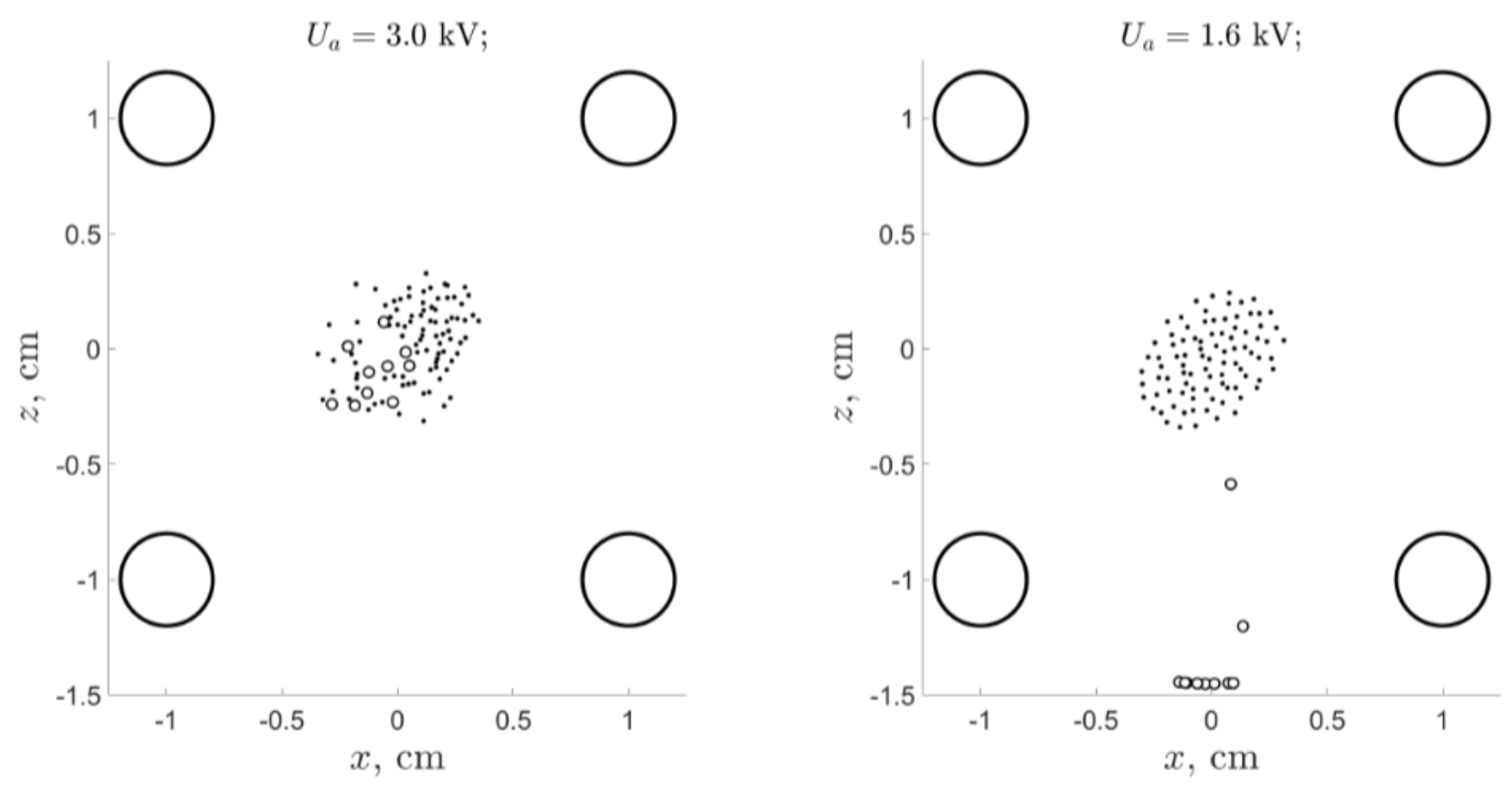

Figure 4: Stimulation of particle behavior in the electrodynamic trap. Left panel: The mixture of charged particles of two types at $U_{a}=3 \mathrm{kV}$ and $\omega=2 \pi \times 50 \mathrm{rad} / \mathrm{s}$ Right panel: particles remaining in the trap when $U_{a}$ was decreased to $1.5 \mathrm{kV}$ 
a) $f=42 \mathrm{~Hz}$

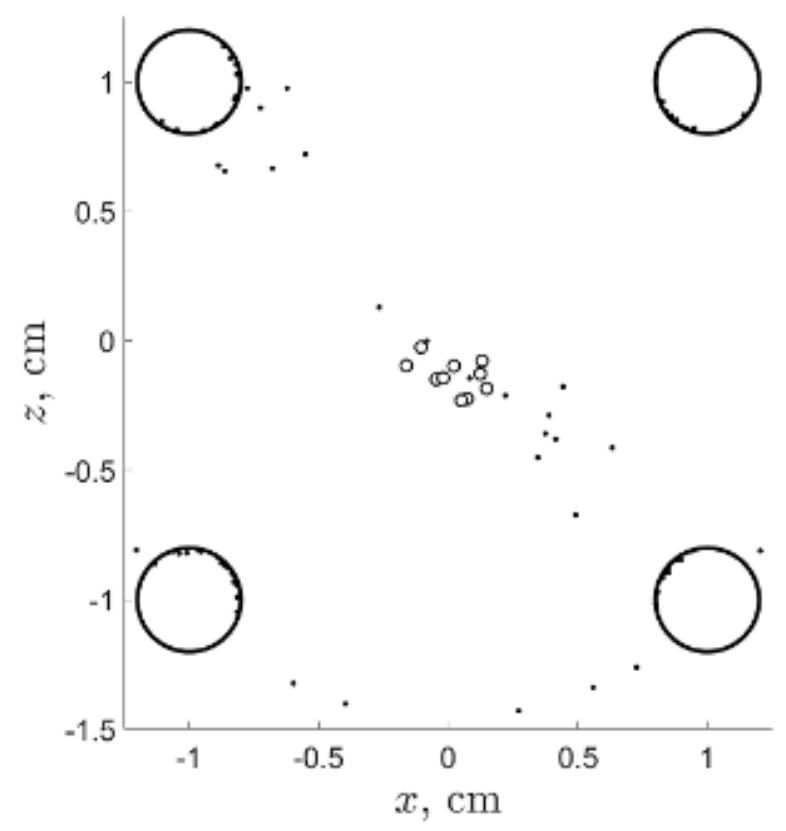

c) $f=150 \mathrm{~Hz}$

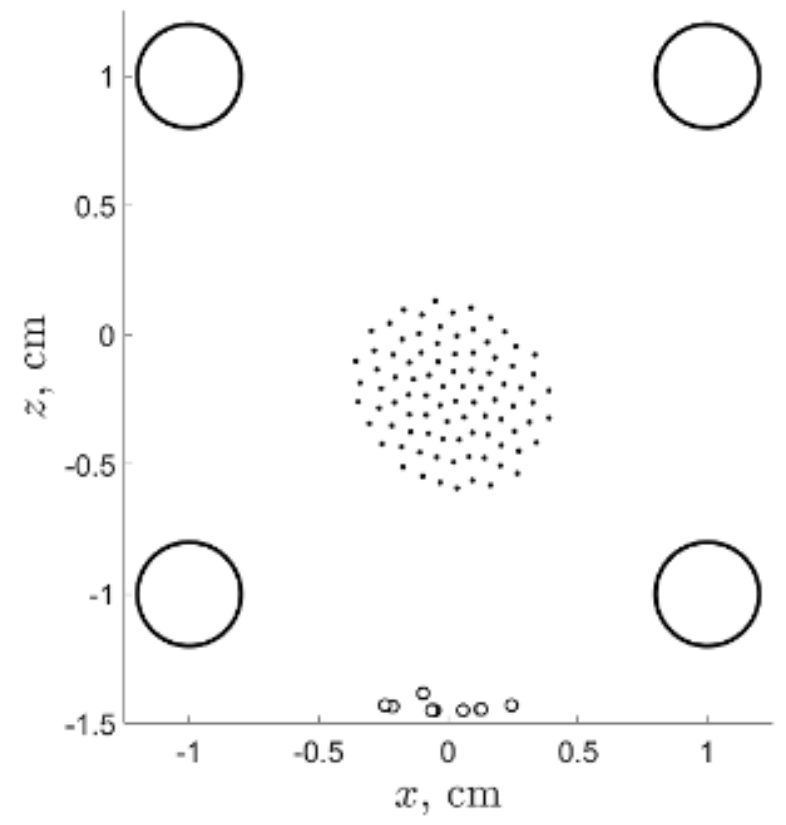

b) $f=50 \mathrm{~Hz}$

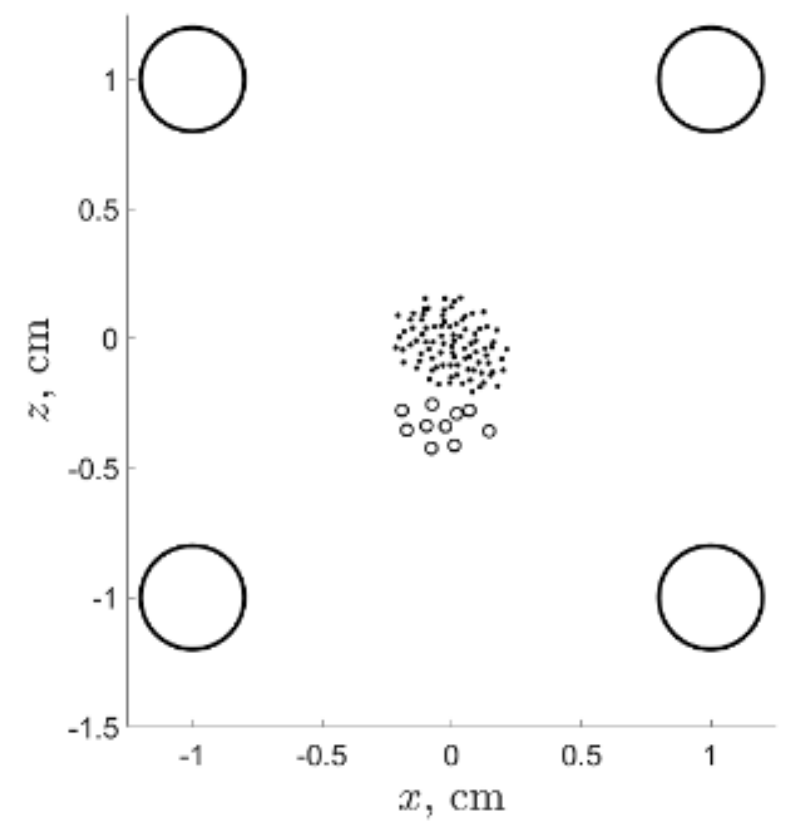

d) $f=190 \mathrm{~Hz}$
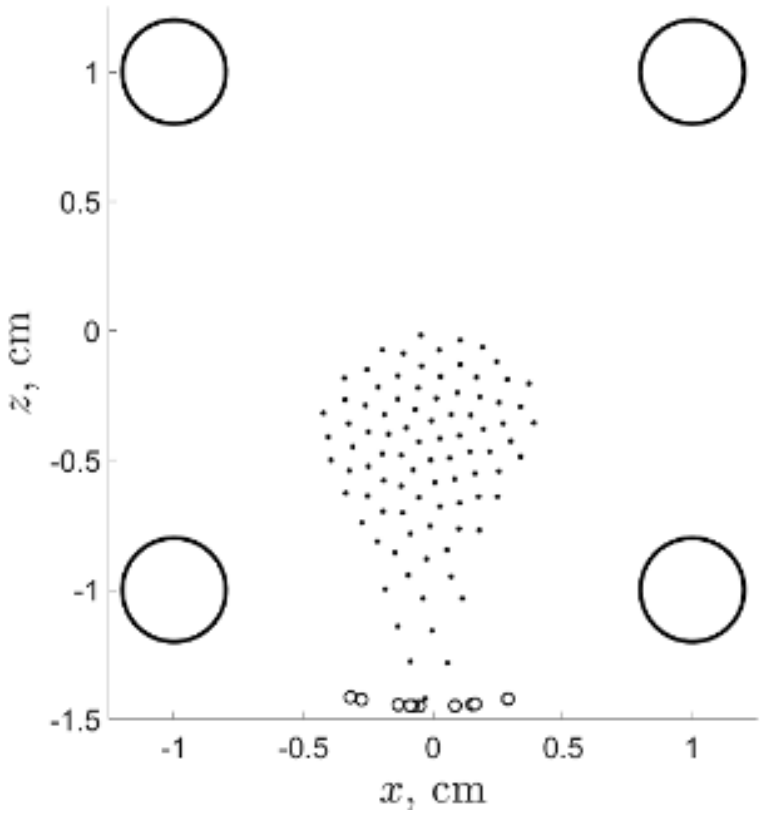

Figure 5: Particle separation and the dynamic behavior of a mixture of two types of particles in the electrodynamic trap at different frequencies of the applied voltage. Circles indicate particles of group 1 with lower $q^{2} / m$ values, dots indicate groups 2 (higher $q^{2} / m$ )

\section{DISCUSSION}

Since the mass of a particle is proportional to its volume, the parameter $\mathrm{q}^{2} / \mathrm{m}$ will be different for particles of different sizes and one can expect the particle separation in an electrodynamic trap. For mixtures with a discrete size distribution function (as in Fig. 5), we can expect the presence of a spatial gap between different particles. It is also possible to ensure that only one sort of particles remain in the trap at varying the frequency.
For polydisperse powders with a continuous distribution function one can expect a gradual increase in the particle size in the dust structure from top to bottom.

Making use of the corona discharge for particle charging has the following peculiarities affected the efficiency of particle separations. In a corona discharge, the charge for particles larger than one micron is determined mainly by the field charging mechanism. In this case, while the particle has not yet acquired a limiting charge, there exist force lines of the electric field crossing its surface. Ions 
moving along these force lines reach the particle surface and charge it. In the case of such charging, the maximum possible charge is determined by the surface area of the particle. It should be noted that for particles less than 0.1 $\mu \mathrm{m}$, the charge is completely determined by the diffusion mechanism. In this case, the charge is proportional to the linear particle size and the difference in $\mathrm{q}^{2} / \mathrm{m}$ for particles of different sizes will be even more significant.

\section{SUMMARY}

It was shown experimentally and numerically that separation of micron-sized particles charged in corona discharge with different sizes occurs in the linear electrodynamic trap due to the different values of $\mathrm{q}^{2} / \mathrm{m}$ for particles of different sizes. By varying the voltage or frequency of the supply voltage, it is possible to achieve that only particles with a larger or smaller value of $\mathrm{q}^{2} / \mathrm{m}$ remain in the trap. It is more conveniently to ensure particles separation by varying the frequency.

\section{ACKNOWLEDGEMENT}

This work was supported by the Ministry of Science and Higher Education of the Russian Federation (State Assignment No. 075-00892-20-01).

\section{REFERENCES}

1. Vishnyakov, V., Dragan, G. (2003). Thermodynamic reasons of agglomeration of dust particles in the thermal dusty plasma. Condensed Matter Physics, vol. 6, 685-692.

2. Fortov, V.E., Ivlev, A.V., Khrapak, S.A., Khrapak, A.G., Morfill, G.E. (2004). Complex (dusty) plasmas: Current status, open issues, perspectives. Physics Reports, vol. 421, 1-103, DOI: 10.1016/j.physrep.2005.08.007

3. Smith, B., Hyde, T., Matthews, L., Reay, J., Cook, M., Schmoke, J. (2008). Phase Transitions in a Dusty Plasma with Two Distinct Particle Sizes. Advances in Space Research, vol. 41, no. 9, 1510-1513, DOI: 10.1016/j.asr.2008.01.006

4. Glushniova, A.V., Saveliev, A.S., Son, E.E., Tereshonok, D.V. (2014). Shock wave-boundary layer interaction on the non-adiabatic ramp surface. High Temperature, vol. 52, no. 2, 220-224, DOI: 10.1134/ S0018151X14020230

5. Privman, V. (2012). Colloids, Nanocrystals, and Surface Nanostructures of Uniform Size and Shape: Modeling of Nucleation and Growth in Solution Synthesis. Sau T.K., Rogach, A.L. (Eds.), ComplexShaped Metal Nanoparticles. John Wiley \& Sons, Ltd, p. 239-268.

6. Singh, M., and Thaokar, R., Khan, A., Mayya, Y. (2018). Theoretical analysis of formation of many-drop arrays in a quadrupole electrodynamic balance. Physical Review E, vol. 98, 032202, DOI: 10.1103/PhysRevE.98.032202
7. Brouwers, B. (1996). Rotational particle separator: A new method for separating fine particles and mists from gases. Chemical Engineering \& Technology, vol. 19, no. 1, 1-10, DOI: 10.1002/ceat.270190102

8. Gascoyne, P.R.C., Vykoukal, J. (2002). Particle separation by dielectrophoresis. Electrophoresis, vol. 23, no. 13, 1973-1983, DOl:10.1002/15222683(200207)23:13<1973::AID-ELPS1973>3.0. $\mathrm{CO} ; 2-1$

9. Xin, H., Bao, D., Zhong, F., Li, B. (2013). Photophoretic separation of particles using two tapered optical fibers. Laser Physics Letters, vol. 10, no. 3, 036004 , DOI: 10.1088/1612-2011/10/3/036004

10. Jonas, A. and Zemanek, P. (2008). Light at work: The use of optical forces for particle manipulation, sorting, and analysis. Electrophoresis, vol. 29, no. 24, 4813-4851, DOI: doi.org/10.1002/elps.200800484

11. Guldiken, R. Jo, M., Gallant, N., Demirci, U., and Zhe, J. (2012). Sheathless Size-Based Acoustic Particle Separation. Sensors, vol. 12, no. 1, 905-922, DOI: $10.3390 /$ s120100905

12. Lapitsky, D.S. (2016). Particle separation by alternating electric fields of quadrupole type. Journal of Physics: Conference Series, vol. 774, 012178, DOI: 10.1088/1742-6596/774/1/012178

13. Libbrecht, K.G., Black, E.D. (2018). Improved microparticle electrodynamic ion traps for physics teaching. American Journal of Physics, vol. 86, no. 7, 539558, DOI: 10.1119/1.5034344

14. Mihalcea, B.M., Giurgiu, L.C., Stan, C., Visan, G.T., Ganciu, M., Filinov, V., Lapitsky, D., Deputatova, L., Syrovatka, R. (2016). Multipole electrodynamic ion trap geometries for microparticle confinement under standard ambient temperature and pressure conditions. Journal of Applied Physics, vol. 119, no. 11, 114303, DOI: 10.1063/1.4943933

15. Stoican, O.S., Mihalcea, B., Viorica Gheorghe (2001). Miniaturized microparticle trapping setup with variable frequency. Romanian Reports in Physics, vol. 53, no. 3-8, 275-280

16. Vasilyak, L., Vladimirov, V., Deputatova, L., Lapitsky, D., Molotkov, V., Pecherkin, V., Filinov, V., Fortov, V. (2013). Coulomb stable structures of charged dust particles in a dynamical trap at atmospheric pressure in air. New Journal of Physics, vol. 15, 043047 , DOI: 10.1088/1367-2630/15/4/043047

17. Syrovatka, R., Filinov V., Vasilyak, L., Fortov, V., Deputatova, L., Vladimirov, V., Pecherkin V. (2019). Solitary density waves in the strongly coupled one component Coulomb particle structures as experimental support of the general versatility of the caustic theory. Physics Letters A, vol. 383, no. 16, 19421945, DOI: 10.1016/j.physleta.2019.03.023 
18. Syrovatka, R., Medvedev, Yu., Filinov, V., Vasilyak, L., Deputatova, L., Vladimirov, V., Pecherkin V. (2019). Solitary waves in a long structure of charged particles confined in the linear Paul trap. Physics Letters A, vol. 383, no. 4, 383-344, DOI: 10.1016/j. physleta.2018.10.044

19. Demyantseva, N.G., Kuzmin, S.M., Solunin, M.A., Solunin, S.A., Solunin, A.M. (2012). On the motion of charged particles in an alternating nonuniform electric field. Technical Physics, vol. 57, no. 11, 14651477, DOI: 10.1134/S1063784212110096

20. Syrovatka, R., Deputatova, L., Filinov, V., Lapitsky, D., Pecherkin, V., Vasiyak, L., Vladimirov, V. (2016). Charge and Mass Measurements of a Dust Particle in the Linear Quadrupole Trap. Contributions to Plasma Physics, vol. 56, no. 5, 419-424, DOI: 10.1002/ ctpp.201500131
21. Lapitsky, D.S., Filinov, V.S., Deputatova, L.V., Vasilyak, L.M., Vladimirov, V.I., Pecherkin, V.Ya. (2013). Dust Particles Behavior in an Electrodynamic Trap. Contributions to Plasma Physics, vol. 53, no. 4-5, 450-456, DOI: 10.1002/ctpp.201300011

22. Guan, W., Joseph, S., Park, J.H., Krstic, P.S., Reed, M.A. (2011). Paul trapping of charged particles in aqueous solution. Proceedings of the National Academy of Sciences, vol. 108, no. 23, 9326-9330, DOI: 10.1073/pnas. 1100977108

23. Park, J.H., Krstic, P.S. (2012). Park J. H., Krstić P. $\mathrm{S}$. Thermal noise in aqueous quadrupole micro-and nano-traps. Nanoscale research letters, vol. 7, no. 1 , 1-13, DOI: 10.1186/1556-276x-7-156 